\title{
Weak Interaction Physics at TwinSol
}

\author{
J. J. Kolata,*, Maxime Brodeur, Tan Ahn, Dan Bardayan, Patrick O’Malley, and J. S. Randhawa \\ ${ }^{1}$ Department of Physics, University of Notre Dame, Notre Dame, Indiana 46556 (USA).
}

\begin{abstract}
A program to investigate the unitarity of the Cabibbo-Kobayashi-Maskawa (CKM) quark-mixing matrix by studying super-allowed mixed mirror $\beta$ decays has been initiated at the TwinSol facility at Notre Dame. These mixed Fermi/Gamow-Teller (F-GT) decays, occurring between $\mathrm{T}=1 / 2$ isospin doublets in mirror nuclei, provide a complimentary check on the data from super-allowed pure Fermi decays from $0^{+}$to $0^{+}$states. The first part of the program, involving the measurement of the lifetimes of the relevant nuclei to the required accuracy of one part in $10^{3}$ or better, has nearly been completed. However, the additional complication introduced by F-GT mixing requires the use of an ion trap to measure the mixing ratio $\rho$ with similar accuracy. The lifetime measurements, as well as progress in installing an ion trap at TwinSol, will be discussed. In addition, since the ion trap will require a dedicated beam line for its operation, an opportunity presented itself to greatly improve the performance of TwinSol for reaction studies with exotic nuclei. This took the form of an added dipole switching magnet coupled to a third solenoid to form the new TriSol facility currently under construction. The expected properties of TriSol, and its application to reaction studies of interest for nuclear astrophysics, will also be discussed.
\end{abstract}

\section{Precision Tests of the Standard Model}

One important test of the Standard Model (SM) of highenergy physics is via the unitarity of the CKM matrix:

$\mathrm{CKM}=\left[\begin{array}{ccc}V_{\mathrm{ud}} & V_{\mathrm{us}} & V_{\mathrm{ub}} \\ V_{\mathrm{cd}} & V_{\mathrm{cs}} & V_{\mathrm{cb}} \\ V_{\mathrm{td}} & V_{\mathrm{ts}} & V_{\mathrm{tb}}\end{array}\right] ;\left|\mathrm{V}_{\mathrm{ud}}\right|^{2}+\left|\mathrm{V}_{\mathrm{us}}\right|^{2}+\left|\mathrm{V}_{\mathrm{ub}}\right|^{2}=1$.

Here, ( $\mathrm{u} \mathrm{d} \mathrm{s} \mathrm{c} \mathrm{b} \mathrm{t)} \mathrm{indicate} \mathrm{the} \mathrm{quarks} \mathrm{of} \mathrm{the} \mathrm{SM.}$ Non-unitarity of this matrix would imply the possibility of physics beyond the SM. The largest element in the summation, $\left|\mathrm{V}_{\mathrm{ud}}\right|^{2}$, is accessible through nuclear $\beta$ decay, and its most precise measurement is via super-allowed pure Fermi decays from $0^{+}$to $0^{+}$states (Figure 1). However, the inferred value must also be accurate so it's important to check it using other techniques. One possibility is via mixed-mirror $\beta$ decay. The required parameters for such a measurement include the half-life, Q-value, branching ratios, and F-GT mixing ratio $\rho$ for the transition. All of these, along with some nuclear correction factors, must be determined with a precision of better than one part in $10^{3}$. A program to determine the relevant half-lives using radioactive beams from TwinSol will be described below, together with progress on construction of an ion trap to measure $\rho$.

\subsection{Half-life measurements}

Half-life measurements have been made for eight $\mathrm{T}=$ $1 / 2$ mixed-mirror transitions; five have been published (Refs. [2-6]) and three more are in preparation [7-9].

*e-mail: jkolata@nd.edu

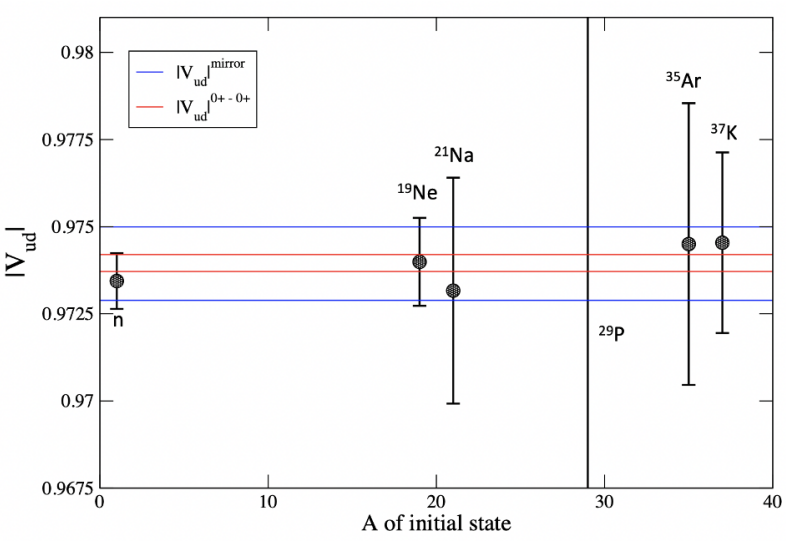

Figure 1. $\left|\mathrm{V}_{\mathrm{ud}}\right|$ from neutron lifetime measurements, $0^{+}$to $0^{+}$ Fermi decay (red error bar), and mixed-mirror decay (blue error bar). Data from Ref. [1], including latest theoretical corrections. The mixed-mirror results agree with those from pure Fermi decay but are a factor of 4.2 less precise.

${ }^{20} \mathrm{~F}$ and ${ }^{28} \mathrm{Al}$, part of a $\mathrm{T}=1$ triplet, have also been studied $[10,11]$. These nuclei can provide information about possible second-class currents $[12,13]$ and also to test the strong form of the conserved vector current (CVC) hypothesis [14].

The experimental apparatus includes the following elements: (1) an Au stopper for the secondary beam was used to eliminate radioactive contaminants formed by the beam incident on impurities in Ta stoppers, (2) the stopper was rotated from the implantation location to the counting location, (3) the primary beam was removed during 


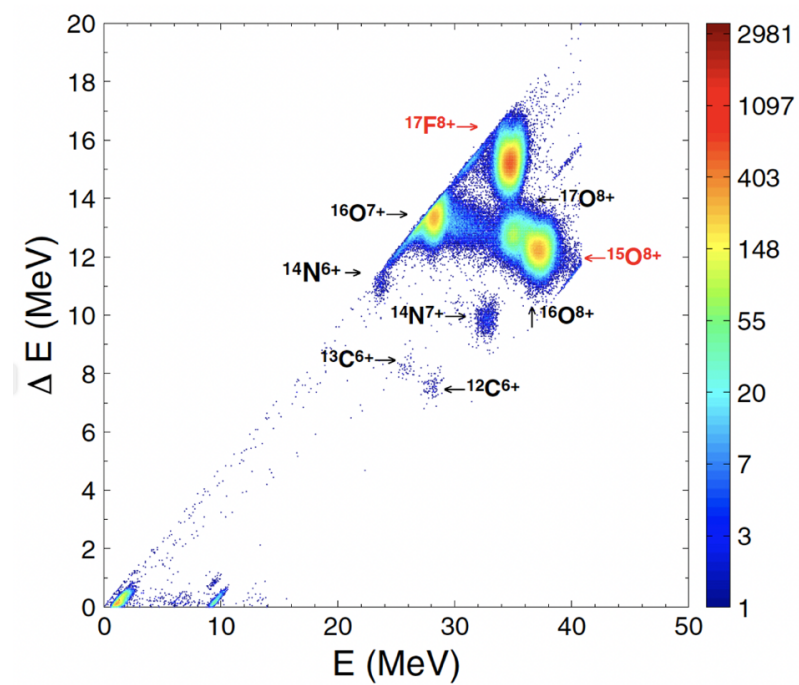

Figure 2. $\Delta \mathrm{E}-\mathrm{E}$ plot for ${ }^{17} \mathrm{~F}$ production, showing a $0.24 \%$ contamination of ${ }^{15} \mathrm{O}$.

the counting period using a pre-tandem electrostatic deflector, (4) the detector was a $1 \mathrm{~mm}$ thick plastic scintillator to reduce $\gamma$-ray background, and (5) a quartz-window photomultiplier detector, which reduced the background by a factor of ten from borosilicate glass windows, was used.

TwinSol typically produces a "cocktail" beam, but it is usually possible to adjust the energy of the primary beam so that only stable ions are produced along with the desired radioactive beam. Nevertheless, small radioactive contaminants, of the order of less than $1 \%$, are sometimes observed (Figure 2). Even such a small contamination can significantly alter the measured half-life when uncertainties of less than $0.1 \%$ are required. Since small contaminant levels cannot always be observed using a Si detector telescope, we also generate a so-called chop plot in each case by removing successively larger amounts of earlytime data and fitting the resulting data set. If there is no contamination, the measured half-life should remain the same until the remaining amount of data is too small to produce statistically significant fits. Such a plot is shown in Figure 3. The contaminant is considered by using a twocomponent fit including its half-life and intensity.

The data were also tested for the presence of very longlived contaminants by fitting with a three-component fit where one of the components had a half-life much greater than that of the nucleus being investigated. It should be noted that all the fits were performed using the so-called "summed fit" procedure described in detail in Ref. [15], which avoids the bias introduced when least-squares fitting is used. Corrections for dead-time were applied, and various systematic errors were estimated. For more details on the analysis, refer to [2-6]. An illustration of the results obtained by these methods in shown in Figure 4. This result, for ${ }^{11} \mathrm{C}$, is the most precise of all superallowed mixed-mirror decays. As the lightest of such systems, ${ }^{11} \mathrm{C}$ provides a laboratory for testing the $\mathrm{CVC}$ hypothesis. See Ref. [4] for further discussion.

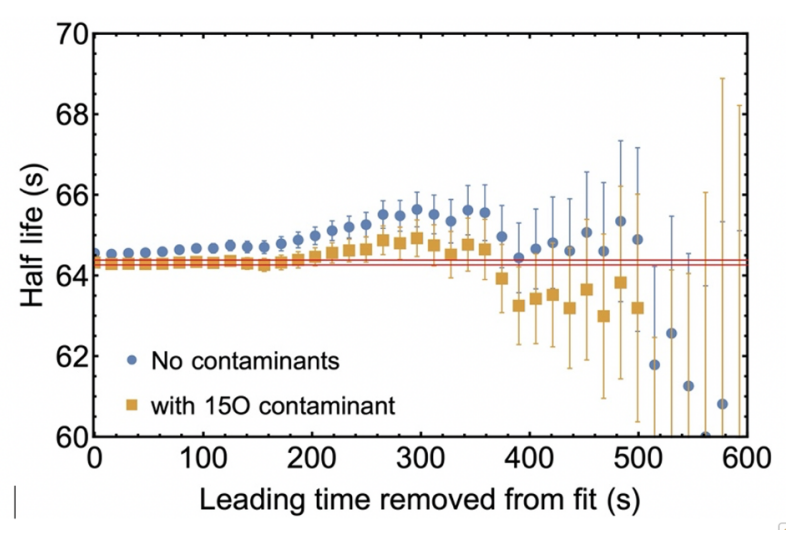

Figure 3. Chop plot for ${ }^{17} \mathrm{~F}$ production showing the improvement obtained by including a $0.2 \%$ contamination of ${ }^{15} \mathrm{O}$.

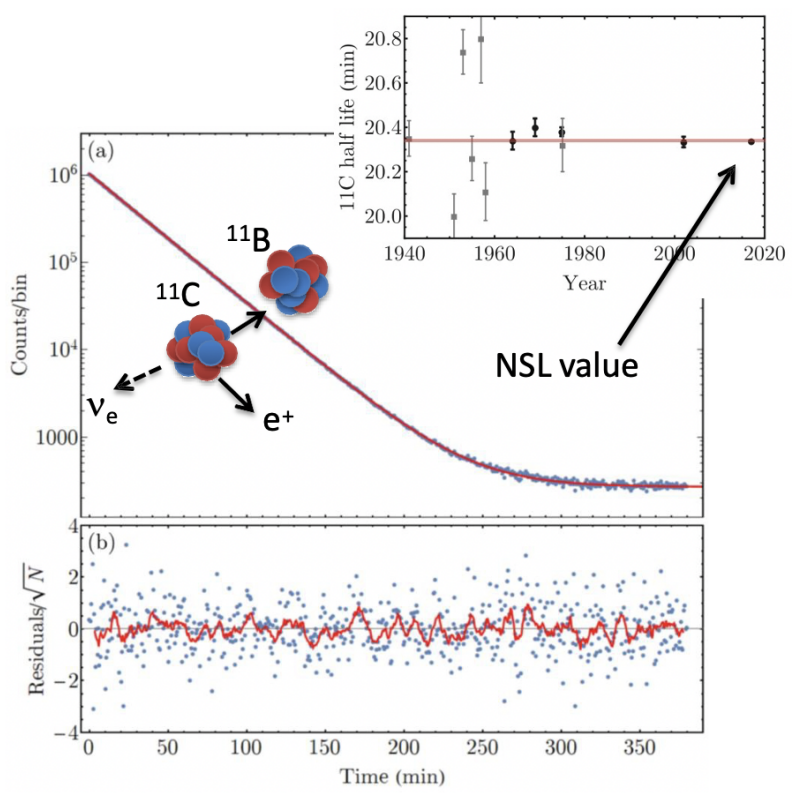

Figure 4. Decay-time plot and fit for ${ }^{11} \mathrm{C}$. The lower portion shows the residuals divided by the square root of the number of counts in the particular bin together with an associated fivepoint running average. The inset shows the measured half-life in various experiments as a function of the year of the measurement.

The fractional uncertainties in $f_{\mathrm{v}}$ values deduced for the relevant mixed-mirror transitions are shown in Figure 5. The eight isotopes measured at Notre Dame are indicated. In all cases except for ${ }^{41} \mathrm{Sc}$ (preliminary analysis) and ${ }^{29} \mathrm{P}$, half-life precision better than one part in $10^{3}$ was achieved.

These are world-average values computed according to criteria from the Particle Data Group [16]. In the case of ${ }^{29} \mathrm{P}$, one existing high-precision measurement could not be excluded, which inflated the error bar on the world average (see Ref. [6] for more details). 


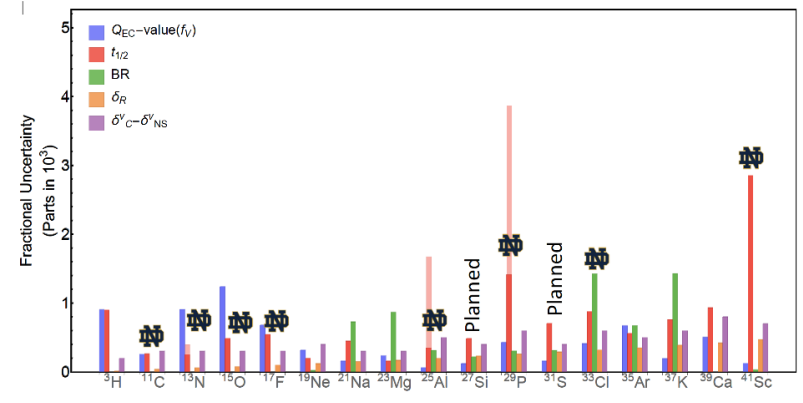

Figure 5. Fractional errors for $f_{\mathrm{v}}$ values of mixed-mirror transitions.

\subsection{Progress on ion-trap commissioning}

As mentioned above, the $f_{\mathrm{v}}$ values alone for mixed-mirror transitions are insufficient to compute $\left|\mathrm{V}_{\mathrm{ud}}\right|$; the F-GT mixing ratio $\rho$ must also be determined with comparable accuracy and precision. There are three methods to determine $\rho$, by measuring: (a) the $\beta$ asymmetry parameter $\mathrm{A}_{\beta}$, (b) the neutrino asymmetry parameter $\mathrm{B}_{v}$, or (c) the $\beta-v$ angular correlation $\mathrm{a}_{\beta v}$ [17]. At present, $\rho$ values have only been obtained with sufficient precision for 5 isotopes (see Figure 1) . There is also data for ${ }^{17} \mathrm{~F}$, but this experiment [18] measured $\mathrm{A}_{\beta}$ which was found to be too insensitive to determine a value for $\left|\mathrm{V}_{\mathrm{ud}}\right|$. In order to add to this data set, the ion trap "St. Benedict" is currently under construction at Notre Dame. A diagram of this system is illustrated in Figure 6 below.

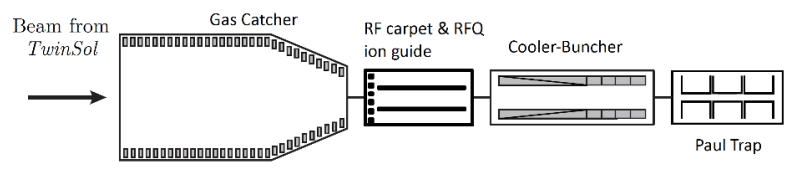

Figure 6. Schematic dagram of the ion trap "St. Benedict".

The gas catcher and cooler-buncher have been completed and tested. Commissioning of the RF carpet and RFQ is currently underway. The first trap experiment will likely be a measurement of $\mathrm{a}_{\beta v}$ for ${ }^{17} \mathrm{~F}$, which is one of the most intense beams $\left(\approx 2 \times 10^{6}\right.$ particles per second $)$ from Twin$\mathrm{Sol}$, and also the most sensitive to possible tensor currents in mirror transitions. Later experiments will focus on ${ }^{15} \mathrm{O}$ and ${ }^{13} \mathrm{~N}$. These light systems are of particular interest since they can also be used to probe for scalar or tensor components in $\beta$ decay.

\section{TriSol}

Since the St. Benedict ion trap requires a dedicated beam line, it was necessary to modify the system in order to preserve the ability to carry out other radioactive beam experiments. This took the form of adding a dipole switching magnet and a third solenoid to form TriSol. The original TwinSol (Figure 7) had its secondary focal point at a distance of about $5.5 \mathrm{~m}$ from the primary target. Under these conditions, the beam spot size was typically $5 \mathrm{~mm}$ full width at half maximum (FWHM). However, in order to enable $\gamma$-ray and neutron experiments, the focal point was shifted to $12 \mathrm{~m}$ so that the beam passed through a $1.25 \mathrm{~m}$ thick high-density concrete shielding wall which basically eliminated background coming from the primary target (see Figure 8). This had two undesirable effects: the diameter of the beam spot was increased to about 25 mm FWHM and the transmission to the secondary target was reduced by about a factor of two. The addition of a third solenoid allowed for the adjustment of the beam emittance in order to greatly improve this situation. The optics were now more complicated, so it was necessary to use the LISE++ program [19] from Michigan State University to optimize the settings of the various elements. A sample beam envelope for a $50 \mathrm{MeV}{ }^{20} \mathrm{Ne}$ beam is shown in Figure 9. The beam spot is now $10 \mathrm{~mm}$ FWHM and its angular divergence is $\pm 1.75^{\circ}$. For lower- $Z$ beams such as ${ }^{6} \mathrm{He}$ or ${ }^{8} \mathrm{~B}$, the spot size is projected to be 5-6 mm FWHM. In addition, there is room behind the third solenoid to move the focal point further upstream to trade spot size for divergence for those experiments that require this.

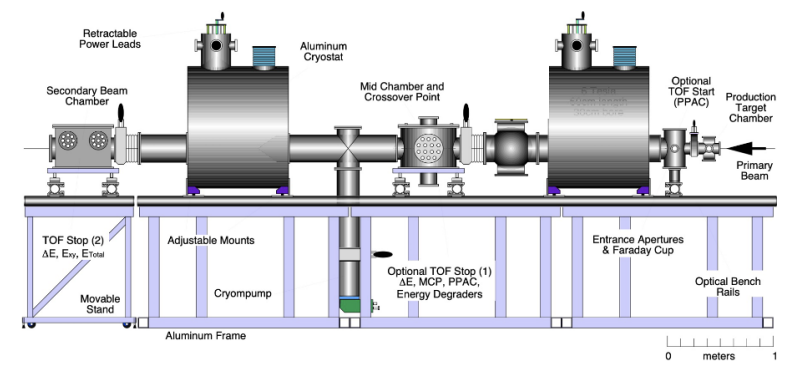

Figure 7. The original TwinSol.

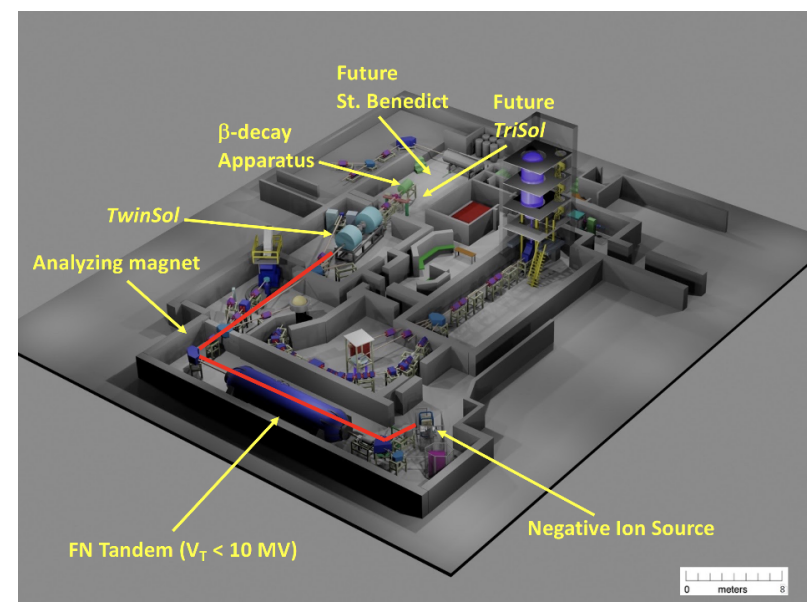

Figure 8. Laboratory diagram showing the location of various elements.

\section{Astrophysical applications of TriSol}

An example of the astrophysically relevant experiments that can be carried out at TriSol is the study of ${ }^{20} \mathrm{Ne}+{ }^{20} \mathrm{Ne}$ fusion cross sections at low energy in order to constrain 


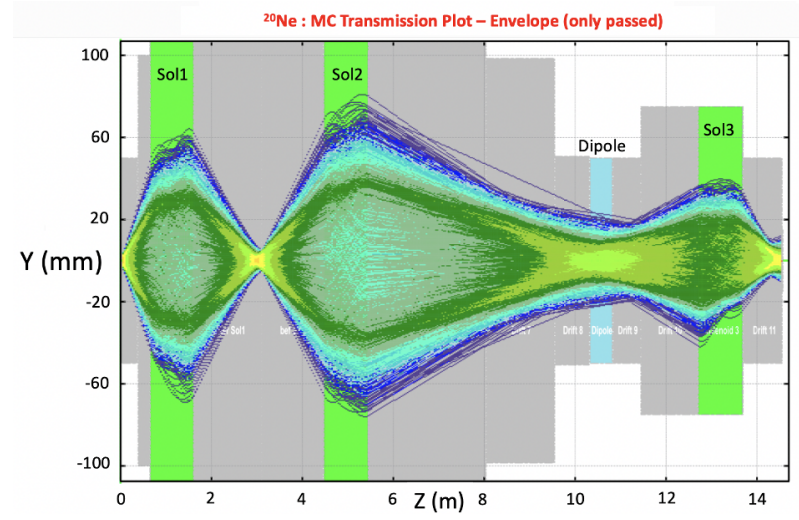

Figure 9. Calculated profile for a $50 \mathrm{MeV}^{20} \mathrm{Ne}$ beam.

pynconuclear burning in accreting neutron stars. A proposal has been submitted to FRIB PAC-1 to measure the ${ }^{26} \mathrm{Ne}+{ }^{20} \mathrm{Ne}$ fusion cross sections using the Active Target Time Projection Chamber (AT-TPC) [20]. A comparison with lighter systems, such as ${ }^{20} \mathrm{Ne}+{ }^{20} \mathrm{Ne}$ and ${ }^{22} \mathrm{Ne}+{ }^{22} \mathrm{Ne}$, is necessary to validate the anticipated results from this experiment. The former of these can be studied at TriSol using the ${ }^{20} \mathrm{Ne}$ beam discussed in the previous section.

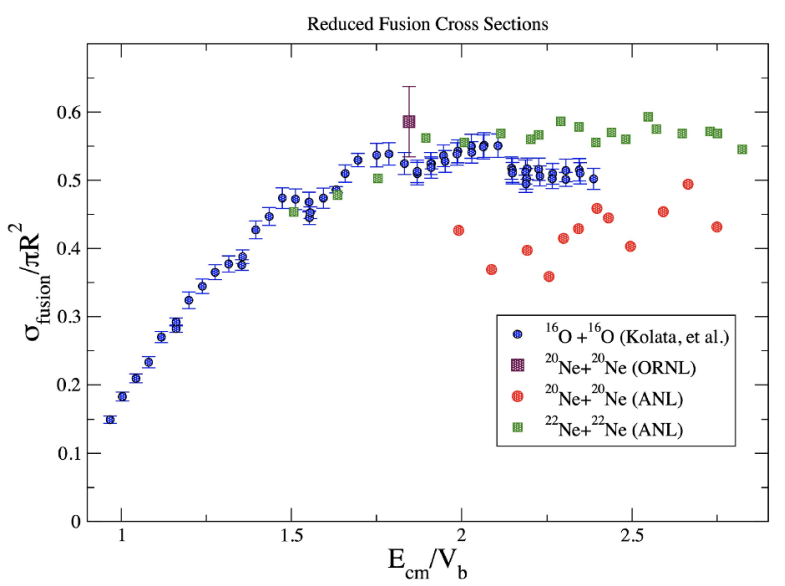

Figure 10. Reduced fusion cross sections for $\mathrm{Ne}+\mathrm{Ne}$ systems. The "ANL" data are from [21] and the "ORNL" data from [22]. A comparison with ${ }^{16} \mathrm{O}+{ }^{16} \mathrm{O}$ fusion [23] is also illustrated.

Existing data on $\mathrm{Ne}+\mathrm{Ne}$ fusion in the region of the Coulomb barrier is illustrated in Figure 10. These are "reduced" fusion cross sections where the effect of nuclear radius $\mathrm{R}$ and barrier $\mathrm{V}_{\mathrm{b}}$ are removed. Here, $\mathrm{R}=1.44\left(\mathrm{~A}_{1}{ }^{1 / 3}+\mathrm{A}_{2}{ }^{1 / 3}\right)$ and $\mathrm{V}_{\mathrm{b}}=1.44\left(\mathrm{Z}_{1} \mathrm{Z}_{2} / \mathrm{R}\right)$ where $\mathrm{R}$ is in $\mathrm{fm}, \mathrm{V}_{\mathrm{b}}$ is in $\mathrm{MeV}$, and $\sigma_{\text {fusion }}$ is in $\mathrm{fm}^{2}$. It can be seen that the ${ }^{20} \mathrm{Ne}+{ }^{20} \mathrm{Ne}$ reduced fusion cross section is considerably smaller than that for the other systems, except possibly for the one data point from Oak Ridge National Laboratory (ORNL).

A more interesting observation is the oscillations that appear in this data, which are similar to the oscillations observed for ${ }^{16} \mathrm{O}+{ }^{16} \mathrm{O}$ occurring due to anti-symmetrization in this identical-particle system. This eliminates odd partial waves; however, it's also necessary for the system to have a "surface-transparent" potential in order to reduce the width of the even-partial-wave "resonances" so they can be observed [23]. The existence of similar oscillations in the ${ }^{20} \mathrm{Ne}+{ }^{20} \mathrm{Ne}$ system, and the hint of similar structure for ${ }^{22} \mathrm{Ne}+{ }^{22} \mathrm{Ne}$, will give important information on the optical-model potentials for these systems if these results can be confirmed.

The role of these data in testing predictions for the astrophysical $\mathrm{S}$-factor for $\mathrm{Ne}+\mathrm{Ne}$ systems is illustrated in Figure 11. The "ORNL" set here includes higher energy data from Ref. [22] and the blue curve is a coupledchannels calculation using the $\mathrm{H} 13$ potential from that reference. The red curve is the prediction from Ref. [24] using the Sao Paulo potential and a polynomial approximation, which becomes increasingly unrealistic above $\mathrm{E}_{\mathrm{cm}}$ $=30 \mathrm{MeV}$.

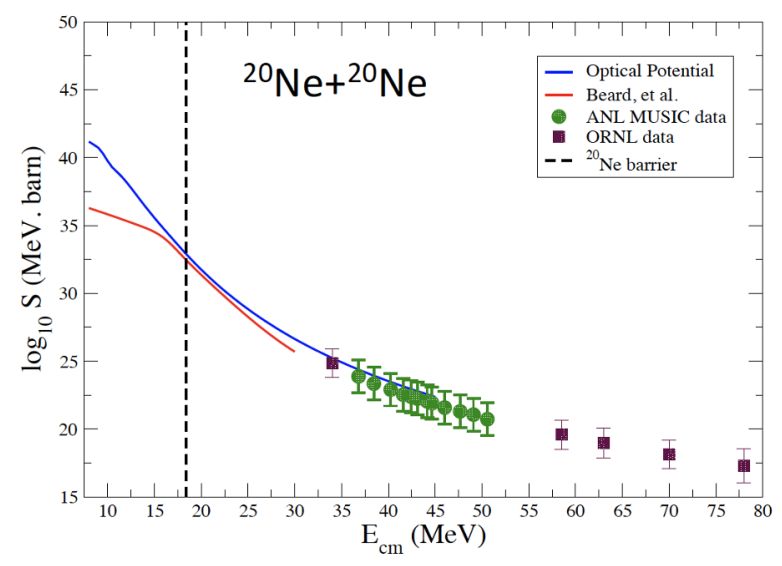

Figure 11. Comparison of the astrophysical S-factor prediction from Ref. [24] with experimental data.

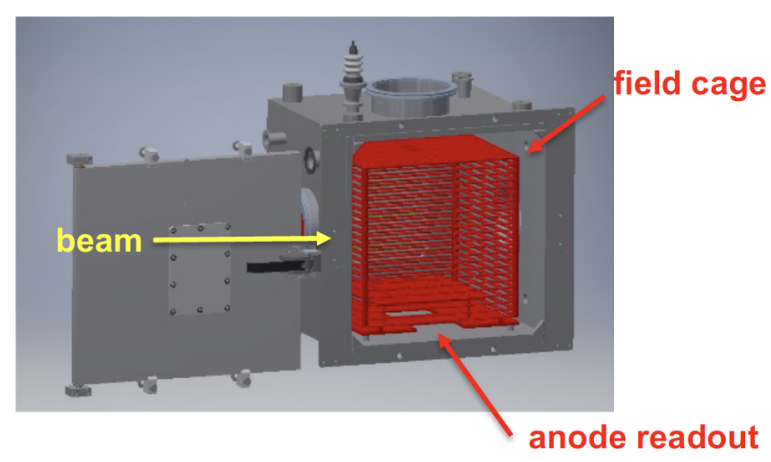

Figure 12. Schematic diagram of the ND-cube time projection chamber

A planned experiment using the $50 \mathrm{MeV}{ }^{20} \mathrm{Ne}$ beam from TriSol should easily reach the region below $\mathrm{E}_{\mathrm{cm}}=16$ $\mathrm{MeV}$ where the disagreement between the Beard, et al. [24] and coupled-channels calculations increases. It will be carried out using the ND-Cube time projection chamber (TPC) [25], illustrated in Fig. 12, which has the advantage of very high efficiency and the ability to measure 
an excitation function over a wide energy range as the incident beam slows down in the target gas which is also the detection medium. The ND-Cube has a rectangular geometry in a $(40 \mathrm{~cm})^{3}$ chamber. The field cage defines a $20 \mathrm{~cm}$ wide by $30 \mathrm{~cm}$ long active area. The drift direction is perpendicular to the pad plane, which is a Micro Pattern Gas Detector (MPGD) having 1000 hexagonal pads. In addition, it incorporates a Micromegas [26] with thick GEM [27] amplification (see [25] for more detail). This detector has an advantage over Multisampling Ionization Chamber (MUSIC) detectors [28] for fusion studies near the barrier due to its large segmentation, which allows for high position resolution near the end of the ion track where the particle energy changes rapidly with the penetration distance. The results of an experiment to measure ${ }^{7} \mathrm{Li}+{ }^{40} \mathrm{Ar}$ fusion cross sections near the barrier is shown in Figure 13. The horizontal error bars indicate the averaging interval used and the vertical error bars are purely statistical. The data are compared with a DWBA prediction using an optical model potential derived from a fit to Li-ion data on a wide range of targets at energies from 13-153 MeV [29]. The result agrees rather well with experiment. This bodes well for future studies of near- and sub-barrier fusion with with the ND-Cube detector.

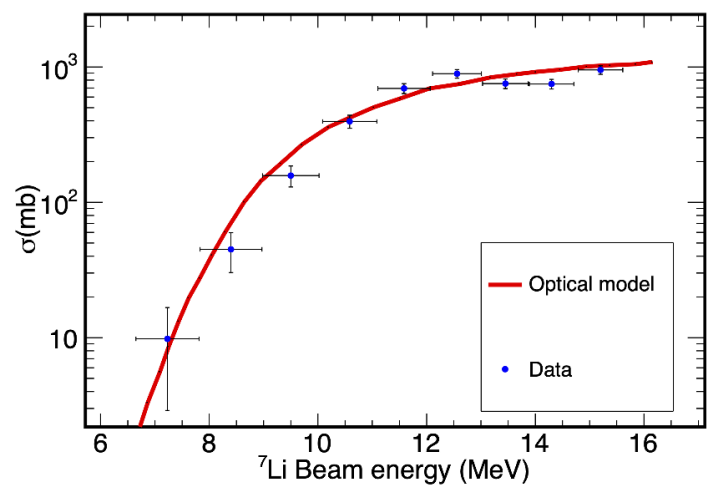

Figure 13. ${ }^{7} \mathrm{Li}+{ }^{40} \mathrm{Ar}$ fusion cross sections near the barrier measured with the ND-Cube TPC. Data taken for 3 hrs. at $\approx 1400$ particles per second, with a P10 gas target.

\section{Acknowledgements}

The weak-interactions collaborators include Drs. Maxime Brodeur, Dan Bardayan, Frederick Becchetti (University of Michigan), James J. Kolata, and Patrick O'Malley together with graduate students Dan Burdette, Biying Liu, Jacob Long, and Adrian Valverde. The TriSol project collaborators include Drs. Tan Ahn, Dan Bardayan, James J. Kolata, Patrick O'Malley and Jaspreet Randhawa together with undergrad student Sydney Coil. Thanks are also due to a number of Notre Dame Nuclear Science Laboratory graduate students who operated the accelerator during the various experiments. This work was partially supported by the U.S. National Science Foundation under Grant Nos. PHY-2011890 and PHY-1725711 (MRI), and by the University of Notre Dame.

\section{References}

[1] Leendert Hayen, Phys. Rev. D 103, 113001 (2021).

[2] M. Brodeur et al., Phys. Rev. C93, 025503 (2016).

[3] J. Long et al., Phys. Rev. C96, 015502 (2017).

[4] A. Valverde et al., Phys. Rev. C97, 035503 (2018).

[5] D. Burdette et al., Phys. Rev. C101, 055504 (2020).

[6] J. Long et al., Phys. Rev. C101, 015501 (2020).

[7] J. Long et al., Phys. Rev. C, submitted $\left({ }^{13} \mathrm{~N}\right)$.

[8] P. O'Malley et al., Phys. Rev. C, in preparation $\left({ }^{33} \mathrm{Cl}\right)$.

[9] B. Liu et al., Phys. Rev. C, in preparation $\left({ }^{41} \mathrm{Sc}\right)$.

[10] D. Burdette et al., Phys. Rev. C99, 015501 (2019).

[11] B. Liu et al., Phys. Rev. C, in preparation $\left({ }^{28} \mathrm{Al}\right)$.

[12] D.H. Wilkinson and D.E. Alburger, Phys. Rev. Lett. 24, 1134 (1970).

[13] N.A. Smirnova and C. Volpe, Nucl. Phys. A 714, 441 (2003).

[14] Nathal Severijns, Marcus Beck, and Oscar NaviliatCuncic, Rev. Mod. Phys. 78, 991 (2006).

[15] V.T. Koslowsky et al., Nucl. Instrum. Methods Phys. Res., Sect. A 401, 289 (1997).

[16] https://pdg.lbl.gov

[17] Nathal Severijns and Oscar Naviliat-Cuncic, Ann. Rev. Nucl. Part. Sci. 61, 23 (2011).

[18] N. Severijns et al., Phys. Rev. Lett. 63, 1050 (1989).

[19] http://lise.nscl.msu.edu/lise.html

[20] Jaspreet S. Randhawa and Rahul Jain, Proposal to FRIB PAC-1, Feb. 12, 2012.

[21] Preliminary data from Argonne National Laboratory, shown with permission from Dr. Melina AvilaCoronado.

[22] D. Shapira et al., Phys. Rev. C28, 1148 (1983).

[23] J.J. Kolata et al., Phys. Rev. C16, 891 (1977).

[24] M. Beard et al., At. Data Nucl. Data Tables 96, 541 (2010).

[25] T. Ahn et al., Nucl. Instrum. Methods Phys. Res., Sect. A (in preparation).

[26] G. Charpak et al., Nucl. Instrum. Methods Phys. Res. Sect. A 478, 26 (2002).

[27] F. Sauli, Nucl. Instrum. Methods Phys. Res. Sect. A 386, 531 (1997).

[28] P. Carnelli et al., Nucl. Instrum. Methods Phys. Res. Sect. A 799, 197 (2015).

[29] J. Cook, Nucl. Phys. A 388, 153 (1982). 